\title{
Supermassive black holes hidden in the outskirts of galaxies
}

\author{
Steffen Mieske \\ European Southern Observatory, \\ Alonso de Cordova 3107, Vitacura, Chile \\ email:smieske@eso.org
}

\begin{abstract}
Ultra-Compact dwarf galaxies, which inhabit the outskirts of galaxies, may conceal a significant population of hitherto overlooked super-massive black holes.
\end{abstract}

Keywords.

Ultra Compact Dwarf Galaxies (UCDs) are pressure supported stellar systems intermediate between dwarf galaxies and star clusters (e.g. Hilker et al. 1999, Drinkwater et al. 2000). Cosmological simulations (e.g. Pfeffer et al. 2016) suggest that a fraction of UCDs are remnants of tidal disruption of intermediate mass $\left(<10^{10} \mathrm{M}_{*}\right)$ galaxy halos. If so, those UCDs should carry a relic of their progenitor galaxy in the form of a massive central black hole. At present this would offset UCDs from the well known $\mathrm{M}_{\mathrm{BH}}-\mathrm{L}$ relation of galaxies (e.g. Graham \& Scott 2013). It is interesting to note that dynamical mass-tolight ratios of UCDs are higher than expected from their stellar populations (e.g. Mieske et al. 2013). This finding is consistent with the hypothesis that supermassive black holes in the centers of UCDs elevate the global velocity dispersion of stars in UCDs (Frank et al. 2011, Mieske et al. 2013). Within that scenario, the measured dynamical M/L ratios suggest super-massive black hole masses of the order of 10-15\% of the UCD mass.

To test the hypothesis that UCDs harbour over-massive central black holes, we targeted with Gemini North NIFS LGS the Virgo cluster UCD M60-UCD1 (Seth et al. 2014). We find that M60-UCD1 has the clear kinematic signature of a supermassive central black hole with $2 \times 10^{7} \mathrm{M}_{*}$, making up $15 \%$ of the UCD mass itself (it also is an X-ray emitter, Strader et al. 2013). This places M60-UCD1 almost 2 dex off the relation between bulge luminosity and central BH mass. Thus our finding indeed suggests that M60-UCD1 is a tidally disrupted remnant of a more massive progenitor of about $10^{10} M_{*}$ ! The next step is to assess whether SMBHs in UCDs are a common feature, thus altering our census of SMBHs in the local universe. To this end we have embarked on a joint Gemini North + VLT campaign targeting about a dozen UCDs, for which data acquisition is ongoing.

\section{References}

Drinkwater, M., et al. 2000, PASA, 17, 227

Frank, M., et al. 2011, MNRAS, 414L, 70

Graham, A. \& Scott, N. 2013, ApJ, 764, 151

Hilker, M., et al. 1999, A\& $A S, 134,75$

Mieske, S., et al. 2013, AछAA, 558A, 14,

Pfeffer, J., et al. 2016, MNRAS, 458, 2492

Seth, A., et al. 2014, Nature, 513, 398

Strader, J., et al. 2013, ApJL, 775, L6 